\title{
OBSCURED ACTIVE GALACTIC NUCLEI
}

\author{
R. Maiolino \\ Osservatorio Astrofisico di Arcetri, Firenze, Italy
}

\begin{abstract}
The properties of the absorption in type 2, narrow line AGNs are reviewed by focusing on the X-ray indicators. I discuss the properties of the cold absorbing medium (the putative torus) and of the reprocessed components, as well as their implications for the unified model. The relation between optical classification and X-ray absorption is examined. The case of "fossil" AGNs, whose type 2 classification is not due to absorption effects, is also discussed. Although this review is mainly focused on nearby Seyfert 2 galaxies, I also shortly discuss the effects of absorption at higher luminosities and higher redshift and the implications for the X-ray background.

KEYWORDS: galaxies: active - galaxies: Seyfert - galaxies: ISM - X-rays: galaxies
\end{abstract}

\section{INTRODUCTION.}

Several observational data indicate that type 2, narrow line Seyfert nuclei suffer significant obscuration along our line of sight. The unified model (Antonucci 1993) ascribes this obscuration to a gaseous parsec-scale circumnuclear torus. According to this model, broad line Seyfert 1 (Sy1) and narrow line Seyfert 2 (Sy2) galaxies would be identical physical objects, while the orientation of the line of sight with respect to the torus is responsible for the obscuration of the Broad Line Region (BLR) and of the nuclear engine (X-UV source) in Sy2s.

In this review I show how X-ray observations, especially at high energies $(>2$ $\mathrm{keV}$ ), provide a wealth of information which constrain the properties of the absorbing medium in Seyfert 2s, i.e. the putative circumnuclear gaseous torus. I also discuss the implications of these X-ray studies on related issues, such as the fuelling of active nuclei and the X-ray background. Due to the limited space, I do not discuss warm absorbers, although evidence for this component is found in some obscured Seyferts (eg. Komossa \& Fink 1997), neither I discuss the soft excess that characterizes most Sy2s (Maiolino et al. 1998, Wilson \& Elvis 1997).

\section{X-RAY ABSORPTION AS A DIAGNOSTIC OF THE TORUS.}

X-ray observations have probably provided the most direct test of the unified model. Indeed, the X-ray spectrum of many Sy2s is characterized by a powerlaw similar to that observed in Sy1s and a photoelectric cutoff due to absorbing, cold gas along our 
line of sight. Since the absorbing column in Sy2s is generally larger than $10^{22} \mathrm{~cm}^{-2}$, these studies were mostly obtained with satellites sensitive in the high energy band above $\sim 1 \mathrm{keV}$ such as Ginga, ASCA and BeppoSAX.

If the absorbing column is larger than $\sim 10^{24} \mathrm{~cm}^{-2}$, i.e. the medium is thick to Compton scattering, then the transmitted component is completely suppressed below $10 \mathrm{keV}$ and the spectrum observed in the $2-10 \mathrm{keV}$ band is dominated by reprocessed components. More specifically, in this case the hard X-ray spectrum is characterized by a flat Compton reflection component, ascribed to the inner surface of the torus, and/or a steeper component due to a ionized, warm scattering medium (eg. Matt et al. 1997). If the absorbing medium has a column in the range $10^{24}-10^{25} \mathrm{~cm}^{-2}$, then the transmitted component can be still observed in the 10 $300 \mathrm{keV}$ band as an emission excess (eg. Done et al. 1996); for larger absorbing columns even the $10-300 \mathrm{keV}$ band is dominated by the reflection component. The cold reflector also produces a prominent fluorescence iron line at $6.4 \mathrm{keV}(\mathrm{EW} \sim 1-2$ $\mathrm{keV}$ with respect to the reflected continuum). Yet, Netzer et al. (1998) pointed out that also the Narrow Line Clouds can contribute significantly to the observed 6.4 $\mathrm{keV}$ line, provided that their column density is large enough. Instead, the warm scattering medium emits lines of $\mathrm{He}-$ and $\mathrm{H}$-like iron at 6.7 and $7 \mathrm{keV}$, which can reach large equivalent widths as well (Matt et al. 1996). Until a few years ago only a couple of Compton thick sources were known. This is because the reflection efficiency of both the cold and warm mirrors is low $\left(\sim 10^{-2}-10^{-3}\right)$ and, therefore, this class of objects is more difficult to detect. This highlights one of the major problems in past hard X-ray surveys, as discussed in the following section.

\subsection{The distribution of $\mathrm{N}_{H}$.}

Past hard X-ray surveys of Sy2s were strongly biased in favor of X-ray bright sources (according to all-sky surveys), which tend to be the least absorbed ones. More recently, X-ray observations have probed X-ray weaker Sy2s, partly removing the selection against heavily obscured objects (eg. Turner et al. 1997). We (Salvati et al. 1997, Maiolino et al. 1998) used BeppoSAX to observe an [OIII]-selected sample of previously unobserved (X-ray weak) Sy2s. These observations discovered a large fraction of Compton thick objects, a result which confirms the bias against heavily obscured systems affecting previous surveys. However, none of these surveys is actually complete or free from biases and, therefore, none of them is suitable (if taken separately) to derive the distribution of absorbing column densities among Sy2s. To accomplish this goal, we merged all the available hard X-ray observations of Sy2s and extracted a complete subsample limited in intrinsic (i.e. unabsorbed) luminosity as inferred from the [OIII] narrow emission line (Risaliti et al. 1999). This subsample is composed of 45 objects and the corresponding $\mathrm{N}_{H}$ distribution, shown in Fig. 1, can be considered the best estimate of the true distribution that can be obtained with the available data. The most interesting result is that this distribution is significantly shifted toward large columns with respect to past estimates: most $(\sim 75 \%)$ of the Sy2s are heavily obscured $\left(\mathrm{N}_{\mathrm{H}}>10^{23} \mathrm{~cm}^{-2}\right)$ and about half are 
Compton thick. The $\mathrm{N}_{H}$ does not appear to correlate with the (intrinsic) luminosity, at variance with early results. This $\mathrm{N}_{H}$ distribution has various implications some of which will be discussed in the following.

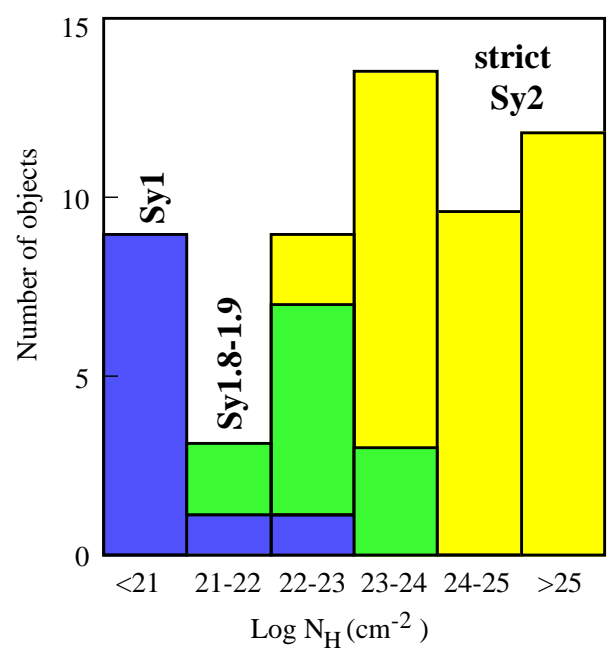

FIGURE 1. The distribution of absorbing column densities among Seyfert galaxies. (from Risaliti et al. 1999, with modifications).

\subsection{X-ray absorption as a probe of the parsec scale torus.}

The large fraction of Compton thick sources constrains the size of the obscuring medium. Indeed, assuming an axisymmetric geometry, the mass of the gas responsible for the obscuration is about $\mathrm{M}_{\text {gas }} \approx f \mathrm{~N}_{\mathrm{H}} \mathrm{R}^{2}$, where $\mathrm{R}$ is the external radius of the torus and $\mathrm{f}$ is the covering factor which, for the Compton thick medium, must be of the order of 0.5. As discussed in Risaliti et al. (1999), the requirement that the gas mass does not exceed the dynamical mass constrains the external radius of the torus to $\mathrm{R}<10$ pc. Enhanced metallicity could help to relax this constraint. Metallicities a few times solar have actually been inferred for the BLR and in the warm absorber of several Sy1s, but an order of magnitude higher metallicities would be required to relax the mass constraints enough to match the size of the torus of a few 100 pc proposed by some models (sect. 2.3).

A parsec-scale size of the obscuring torus is also supported by the variability of $\mathrm{N}_{H}$. Indeed, in several Seyfert galaxies the photoelectric cutoff is observed to change significantly on time scales of a few years (Malizia et al. 1997). Fig. 2 shows the specific case of NGC7582 (from a compilation of results in Turner et al. 2000, Xue et al. 1999 and references therein, where the same model was adopted to fit the data). Although in some cases differences in $\mathrm{N}_{H}$ measured at different epochs might be ascribed to the different satellite used, in some cases variation of $\mathrm{N}_{H}$ are observed even with the same satellite. Finally, I recall that a parsec-scale component of the obscuring torus has been directly observed in radio VLBI images, both in continuum (free-free) and $\mathrm{H}_{2} \mathrm{O}$ maser emission, in a few active nuclei (eg. Gallimore 
et al. 1997, Greenhill \& Gwinn 1997).

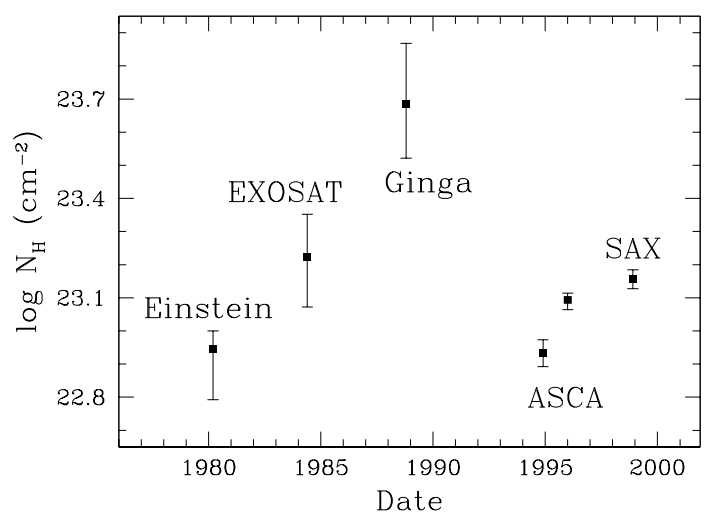

FIGURE 2. Variation of the absorbing $\mathrm{N}_{H}$ in the Seyfert 2 galaxy NGC7582.

2.3. X-ray absorption as a probe of the $100 \mathrm{pc}$ scale torus.

$\mathrm{X}$-ray studies have also provided evidence for obscuring medium on larger scales $(\sim 100 \mathrm{pc})$. Indeed, samples of AGNs selected in the soft X-rays $(\leq 2 \mathrm{keV})$ are characterized by a shortage of edge-on galaxies (Lawrence \& Elvis 1982, McLeod \& Rieke 1995, Simcoe et al. 1997). This effect is ascribed to circumnuclear material, associated to the galactic gaseous disk, which obscures the AGN when the host galaxy is oriented edge-on. Only a moderate absorbing column $\left(\mathrm{N}_{\mathrm{H}} \geq 10^{22} \mathrm{~cm}^{-2}\right)$ is required to suppress significantly the soft X-ray emission.

A $100 \mathrm{pc}$ scale component of the obscuring torus is also required to fit the broad IR spectral energy distribution (Granato et al. 1997) and it is also directly observed in the HST images of some objects (eg. Ford et al. 1997, Malkan et al. 1998).

\subsection{Intermediate type Seyferts.}

Very likely, the obscuring torus has both a Compton thick parsec-scale component and a larger $100 \mathrm{pc}$-scale component which is coplanar with the galactic disk and characterized by lower $\mathrm{N}_{H}$ (which does not violates the mass constraints). The internal torus obscures completely the BLR along the intercepting lines of sight (Sy2 case), while the obscuration due to the external torus (in edge-on systems) is moderate and the Seyfert nucleus might show weak broad lines and, therefore, might be classified as intermediate type 1.8-1.9. Indeed, intermediate type Sys are more commonly found in edge-on galaxies (Maiolino \& Rieke 1995). This model is also supported by the distribution of absorbing column of intermediate Seyferts, which is significantly shifted towards lower values of $\mathrm{N}_{H}$ than strict Sy2s (Risaliti et al. 1999, Fig. 1). Although, this model might apply to many intermediate type Seyferts, there are Seyfert galaxies whose intermediate properties are not to ascribe to moderate obscuration but to photoionization and variability effects or to more complex forms of absorption (Goodrich et al. 1995, Komossa \& Fink 1997). 


\subsection{Dual absorbers.}

A simple, absorbed power law does not provide an adequate description of the observed X-ray spectrum for some of the Compton thin Sy2s. In these cases there is evidence for a second photoelectric cutoff at higher energies due to a medium that only partially covers the X-ray source (Malaguti et al. 1999, Hayashi et al. 1996, Weaver et al. 1994, Vignali et al. 1998). While the photoelectric cutoff at low energies is very likely due to the torus, the partial covering observed at higher energies can only be obtained if the second absorbing medium is close to the X-ray source and has a similar size. A most likely candidate is the BLR. Generally, the column density of the partial covering medium is of the order of $\sim 10^{23} \mathrm{~cm}^{-2}$, in agreement with estimates for the $\mathrm{N}_{H}$ of broad line clouds. The covering factor of the partial absorber is generally found to be larger than $\sim 30 \%$, that is significantly higher than the covering factor of the broad line clouds which, based on the broad lines equivalent width and on the absence of the Ly-edge cutoff in the UV spectrum of QSOs, is expected to be about $10 \%$. However, I do not consider this a major caveat since the dual absorbers discovered so far are not representative of the true distribution of the partial covering absorption systems, but only sample the tail with high covering factor. Partial covering systems with a covering factor of $10 \%$ probably remained undetected in the past observations. I expect Chandra and XMM to discover a large number of dual absorbers with low partial covering.

Finally, thanks to the extended spectral coverage of BeppoSAX some cases of dual absorber with partial covering characterized by an absorbing column as high as $\mathrm{N}_{\mathrm{H}} \sim 10^{24} \mathrm{~cm}^{-2}$ are being found (eg. Turner et al. 2000). Such a high $\mathrm{N}_{H}$ is still consistent with that expected for the broad line clouds. Indeed, the estimated column of $10^{22}-10^{23} \mathrm{~cm}^{-2}$ for the broad line clouds is only a lower limit that is required to produce the low ionization broad emission lines (MgII, FeII, etc...).

\section{X-RAY ABSORPTION AND GALAXY MORPHOLOGY.}

Although the column density of the cold obscuring material does not depend on the properties of the AGN (eg. its luminosity) it does depend on the properties of the host galaxy. Indeed, the absorbing $\mathrm{N}_{H}$ strongly correlates with the presence of a stellar bar in the host galaxy (Maiolino et al. 1999). In particular, while non-barred Sy2s are characterized by an average $\log \mathrm{N}_{\mathrm{H}} \sim 22\left(\mathrm{~cm}^{-2}\right)$, most of the strongly barred Sy2 galaxies are Compton thick. This finding indicates that stellar bars are effective in driving gas into the nuclear region to obscure the AGN. This result is in line with other studies, as reviewed by Sakamoto (1999), which indicate that bars are effective in driving gas into the nuclear region, though these other studies are not specifically focused on AGNs. We might speculate that, more generally, nonaxisymmetric potentials (eg. distorted morphologies and galaxy interactions) drive gas into the nuclear region. Although there are some observational indications in this direction, a systemic study, similar to that on barred systems, has not been performed yet. This will be possible with the new Chandra and XMM data. 


\section{THE RELATION BETWEEN OPTICAL AND X-RAY ABSORPTION.}

If the obscuring torus has the same gas-to-dust ratio as in the Galactic ISM, and the dust is characterized a Galactic extinction curve, then the nuclear region of Sy2s should suffer a visual extinction that is related to the gaseous column density by the formula $A_{V}=5 \times 10^{-22} \mathrm{~N}_{\mathrm{H}}\left(\mathrm{cm}^{-2}\right)$. In general this is not the case: $A_{V}$ is lower than expected from the $\mathrm{N}_{H}$ measured in the X-rays. This was first pointed out by Maccacaro et al. (1982). A visual extinction lower than that expected from the $\mathrm{N}_{H}$ measured in the X-rays is also required to fit the IR spectrum of AGNs (Granato et al. 1997). We have collected a sample of Seyferts which both show X-ray (cold) absorption and whose optical or IR broad lines are not completely suppressed. The ratios between the broad lines provide information on the dust reddening towards the nucleus; however, the broad emission lines must be used with much care, since the extreme conditions of the broad line clouds can affect the intrinsic line ratios through radiative transport effects. By assuming the standard extinction curve we can estimate the visual extinction. The resulting distribution for the $A_{V} / N_{H}$ ratio, relative to the Galactic standard value, is shown in Fig. 3. Most of the AGNs in our sample are characterized by a deficit of dust absorption with respect to what expected from the $\mathrm{N}_{H}$ measured in the X-rays, in agreement with early claims. At higher, quasar-like luminosities there are even more extreme examples of this effect: objects that, although absorbed in the X-rays, do not show significant dust absorption in the optical and appear as type 1, broad line AGNs have been recently discovered in hard X-ray and radio surveys (Sambruna et al. 1999, Akiyama et al. 2000, Reeves et al. 1997). Puzzling enough, the early Chandra surveys presented to date have found only a few type 1 QSOs absorbed in the hard X-rays; this issue will be shortly discussed in Sect. 7 .

The origin of the reduced $A_{V} / N_{H}$ ratio is not clear. An obvious explanation is that the dust-to-gas ratio is much lower than Galactic or that in the inner part of the obscuring torus the dust is sublimated by the strong UV radiation field. However, if the dust content in the absorbing medium is significantly reduced, especially at the inner face, then most of the UV ionizing photons are absorbed by the atomic gas. This should create a huge HII region, which would emit strong ( narrow) hydrogen lines corresponding to a large covering factor, i.e. much brighter than the emission lines from the NLR (see also Netzer \& Laor, 1993). Also, a simple shortage of dust grains with respect to the gas mass would not explain other peculiar properties of the dust in AGNs, such as the absence of the silicate absorption feature in the mid-IR spectra of most Sy2s (Clavel et al. 2000) and the absence of the carbon dip in the UV spectra of some reddened Sy1s.

Another interesting possibility is that the dust extinction curve is much flatter than the standard Galactic. The high density of the gas in the circumnuclear region of AGNs is likely to favor the growth of large grains (probably through coagulation) which, in turn, should flatten the extinction curve and make it featureless. This effect is directly observed in the dense clouds of our Galaxy (Draine 1995). Within the context of the optical versus X-ray absorption, the effect of a flat extinction curve 
(due to grain coagulation) is twofold: 1) given the same dust mass, the effective visual extinction is lower, and 2) the broad lines ratio gives a deceiving (low) measure of the extinction. A more thorough discussion of the whole issue is given in Maiolino et al. (2000b).

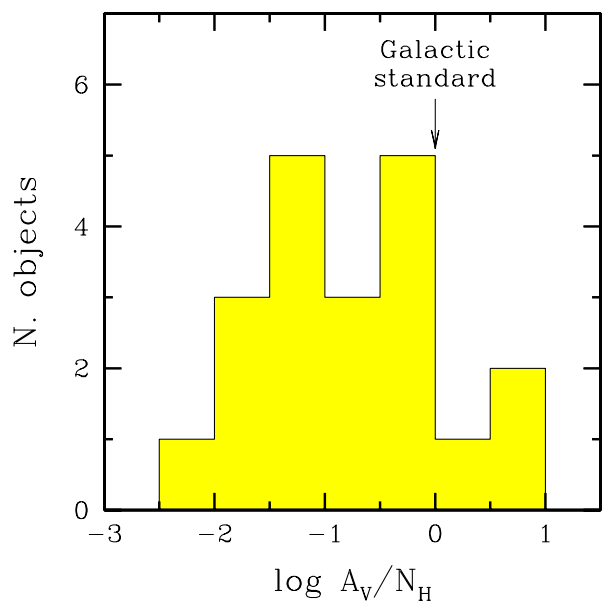

FIGURE 3. Distribution of the $\mathrm{A}_{\mathrm{V}} / \mathrm{N}_{\mathrm{H}}$ ratio, relative to the Galactic standard value, for a sample of absorbed AGNs.

\section{PIERCING COMPLETELY HIDDEN AGNS.}

So far I have discussed X-ray absorption in AGNs which were discovered and classified in the optical. However, an increasing number of obscured powerful AGNs has been discovered by means of hard X-ray observations in galaxies which are optically classified as starburst or LINER. Probably, in these objects the obscuring medium hides also the NLR. Alternatively, the nuclear ionizing source might be completely embedded and obscured in all directions. The most spectacular case is the nearby (4 Mpc) edge-on galaxy NGC4945, which hosts one of the brightest AGNs at $100 \mathrm{keV}$ (Done et al. 1996) The powerful X-ray nucleus is obscured by a column of $\sim 5 \times 10^{24} \mathrm{~cm}^{-2}$ along our line of sight. However, it seems that the nucleus is heavily obscured in all directions. Indeed, optical to mid-IR observations were unable to detect any indication of the AGN activity: at these wavelengths the central region is characterized by a starburst with a spectacular superwind cavity where LINER-like lines are produced (Maiolino et al. 2000a, Marconi et al. 2000). A case quite similar to NGC4945, but at much higher luminosities, is NGC6240. The optical spectrum of this strongly interacting system shows only weak LINER-like emission lines and the mid-IR properties are similar to starburst galaxies (Lutz et al. 1999). However, hard X-ray observations have detected the presence of a heavily obscured AGN whose intrinsic luminosity is in the QSO range (Vignati et al. 1999). Other luminous IR galaxies, optically classified as starburst or LINER, might also host completely hidden AGNs (Risaliti et al. 2000).

Some of the luminous hard X-ray sources recently discovered by deep Chandra 
surveys have optical and near-IR counterparts that show little or no evidence for AGN activity (eg. Mushotzky et al. 2000, Fiore et al. 2000). Possibly, many of these objects are the analogous at higher redshift of the local, completely hidden AGNs discussed above.

\section{FOSSIL ACTIVE NUCLEI.}

Not all of the narrow line, type 2 Seyfert galaxies are associated to absorption along our line of sight. A fraction of the Seyfert galaxies have shown a strong decrease of their X-ray flux, on time scales of several years, that is not associated to an increased absorption but to an intrinsic drop of their activity (eg. Bassani et al. 1999b). One of the best studied cases is NGC2992 (Weaver et al. 1996). The fraction of these "fossil" AGNs is about 10\% (Maiolino et al. in prep.). However, this is actually a lower limit to their real fraction since the identification of this class of objects requires X-ray observations at different epochs and, in particular, the source had to be in a high, bright state during the observations performed by the early, low sensitivity missions. These conditions are met only for a limited number of Seyferts. Obviously, the conservation of the number of active nuclei requires fossil AGNs to revive after a certain period, as it is actually observed (eg. Gilli et al. 2000a).

Shortly after that an AGN turns off, the BLR fades and the nucleus appears as a type 2 Seyfert whose X-ray emission is dominated by the cold reflection component due to the circumnuclear torus (Guainazzi et al. 1998). Although the AGN appears as a Compton thick Sy2, these features are not due to obscuration. About 10 years later also the echo from the torus should fade. If the nucleus remains in a quiescent state for an even longer period then also the gas in NLR clouds can recombine. Yet, the high ionization regions of the clouds should recombine much faster and, therefore, the observed narrow line spectrum should be characterized by low ionization lines similar to LINERs (Eracleous et al. 1995). In this phase the X-ray emission should be dominated by the non-variable warm reflection component with, possibly a highly ionized Fe line at $6.7-7 \mathrm{keV}$, as indeed observed in several LINERs. Some of the LINER nuclei might actually be fossil AGNs.

\section{IMPLICATIONS FOR THE X-RAY BACKGROUND.}

Obscured AGNs are thought to be a key ingredient of the hard X-ray background (XRB, Setti \& Woltjer 1991, Comastri et al. 1995). This is discussed in detail in this volume by other authors (eg. Comastri). However, I wish to emphasize that some of the issues discussed in this review might have implications for our understanding of the origin of the hard XRB.

The distribution of $\mathrm{N}_{H}$ represents the main set of free parameters in the XRB synthesis models. The $\mathrm{N}_{H}$ distribution presented in sect. 2.1 can be used to freeze this set of parameters, under the assumption that the distribution does not evolve with redshift. Detailed models that take into account this constraint are presented in Gilli et al. (1999, 2000b): although the shape and power of the XRB is well 
reproduced, the observed number counts in the hard X-rays seem to require an evolution of the obscured AGNs faster than for the unobscured population. This result is also related to the finding that non-axisymmetric morphologies increase the obscuration of the active nuclei (sect. 3). Indeed, the latter effect should have an impact on the XRB, since galaxies at high redshift are characterized by a higher rate of distorted/irregular morphologies.

The deficiency of dust absorption, with respect to the X-ray absorption, especially at high luminosities, implies a possible mismatch between optical and X-ray classification of the sources contributing to the hard X-ray background. In particular, some of the type 2 QSOs, which are expected to make most of the hard XRB, could be optically "masked" as type 1 QSOs and already present in optical surveys. As mentioned in Sect. 4, the early Chandra surveys have identified only a few objects of this class, at variance with what found in radio and past hard $\mathrm{X}$-surveys (Sect. 4). Possibly the discrepancy is due to the sensitivity of Chandra which peaks in the soft X-ray band and, therefore, probably biases the surveys in favor of little absorbed sources. To properly tackle this issue we should wait for the identification of a larger number of Chandra sources (especially at fainter fluxes, where the fraction of absorbed sources is higher) and the results of the surveys that are being performed with XMM, whose sensitivity is much more uniform up to $\sim 7 \mathrm{keV}$.

\section{ACKNOWLEDGEMENTS}

Many of the new results presented in this paper were obtained in collaboration with R. Gilli, A. Marconi, G. Risaliti and M. Salvati. This work was partially supported by the Italian Space Agency (ASI) through the grant ARS-99-15 and by the Italian Ministry for University and Research (MURST) through the grant Cofin-98-02-32.

\section{REFERENCES}

Antonucci, R.R.J., 1993, ARA\&A 31, 473

Akiyama, M., et al., 2000, ApJ, 532, 700

Bassani, L., et al. 1999b, ApJS, 121, 473

Clavel, J., et al., 2000, A\&A, 357, 839

Comastri, A., Setti, G., Zamorani, G., Hasinger, G., 1995, A\&A, 296, 1

Done, C., Madejski, G.M., Smith, D.A., 1996, ApJ, 463, L63

Draine, B.T., 1995, in The physics of the interstellar medium and intergalactic medium, ASP Conf. Ser. 80, p.133

Eracleous, M., Livio, M., Binette, L., 1995, ApJ 445, L1

Fiore, F., et al., 2000, New Astr. in press astro-ph/0003273

Ford, H., et al., 1997, in IAU Coll. 163, ASP Conf. Ser. 121, p.620

Gallimore, J.F., Baum, S.A., O‘Dea, C.P., 1997, Nat., 388, 852

Gilli, R., Risaliti, G., Salvati, M., 1999, A\&A, 347, 424

Gilli, R., et al. 2000a, A\&A, 355, 485

Gilli, R., 2000b, in "Fourth Italian Conference on AGNs", MemSAIt, in press, astro-ph/0007207) 
Goodrich, R.W., 1995, ApJ, 440, 141

Granato, G.L., Danese, L., Franceschini, A., 1997, ApJ, 486, 147

Greenhill, L.J., Gwinn, C.R., 1997, Ap\&SS, 248, 261

Guainazzi, M, et al., 1998, MNRAS, 301, L1

Hayashi, I., Koyama, K., Awaki, H., 1996, PASJ, 48, 219

Iwasawa, K., 1999, MNRAS, 302, 96

Komossa, S., Fink, H., 1997, A\&A 327, 555

Lawrence, A., Elvis, M., 1982, ApJ, 256, 410

Lutz, D., Veilleux, S., Genzel, R., 1999, ApJ, 517, L13

Maccacaro, T., Perola, G.C., Elvis, M., 1982, ApJ, 257, 47

Malaguti, G., et al., 1999, A\&A, 342, L41

Malizia, A., et al. 1997, ApJ 113, 311

Maiolino, R., Rieke, G.H., 1995, ApJ, 454, 95

Maiolino, R., et al., 1998, A\&A, 338, 781

Maiolino, R., Risaliti, G., Salvati, M., 1999a, A\&A, 341, L35

Maiolino, R., et al. 2000a, in Imaging the Universe in Three Dimensions, Eds. W. van Breugel \& J. Bland-Hawthorn, ASP Conf. Ser. 195, p. 307 astro-ph/9906038)

Maiolino, R., et al. 2000b, A\&A submitt

Malkan, M.A., Gorjian, V., Tam., R., 1998, ApJS, 117, 25

Marconi, A., et al., 2000, A\&A, 357, 24

Matt, G., Brandt, W.N., Fabian, A.C., 1996, MNRAS, 280, 823

Matt, G., et al. 1997, A\&A, 325, L13

McLeod, K.K., Rieke, G.H., 1995, ApJ, 441, 96

Mushotzky, R.F., Cowie, L.L., Barger, A.J., Arnaud, K.A., 2000, Nat, 404, 459

Netzer, H., Laor, A., 1993, ApJ, 404, 51

Netzer, H., Turner, T.J., George, I.M., 1998, ApJ, 504, 680

Reeves, J.N., Turner, M.J.L., Ohashi, T., Kii, T., 1997, MNRAS, 292, 468

Risaliti, G., Maiolino, R., Salvati, M., 1999, ApJ, 522, 157

Risaliti, G., Gilli, R. Maiolino, R., Salvati, M., 2000, A\&A, 357, 13

Sakamoto, K., 1999, in Galaxy Dynamics: from Early Universe to the Present, ASP Conf. Ser., in press astro-ph/9910226)

Salvati, M., et al., 1997, A\&A, 323, L1

Sambruna, R.M., Eracleous, M., Mushotzky, R.F., 1999, ApJ, 526, 60

Setti, G., Woltjer, L., 1989, A\&A, 224, 21

Simcoe, R., McLeod, K.K., Schachter, J., Elvis, M., 1997, ApJ, 489, 615

Turner, T.J., et al., 2000, ApJ, 531, 245

Vignali, C., et al., 1998, A\&A, 333, 411

Vignati, P., et al., 1999, A\&A, 349, L57

Weaver, K.A., et al. 1996, ApJ, 458, 160

Wilson, A.S., Elvis, M., 1997, Ap\&SS, 248, 141

Xue, S.-J., et al., 1998, PASJ, 50, 519 